\title{
Charges and Fluxes for Branes
}

\section{David Olive*}

University of Wales Swansea

E-mail: D.I.Olive@Swansea.ac.uk

ABSTRACT: Branes coupling geometrically to abelian gauge fields within a fixed spacetime of any dimension and topology are considered with a view to determining the various types of conserved quantity, charges and fluxes, electric and magnetic. The occurrence of non-zero electric charges requires space-time to be open rather than closed. The resultant classification theory involves relative homology and not just absolute homology. An important mathematical property is the exact sequence of homology/cohomology and it has a vital physical interpretation

String theory now seems to constitute the culmination of a long history of ideas concerning the unification of forces. About thirty years ago it was discovered that the consistent quantisation of strings compatible with relativity required space-time to have a dimension of 10 or 26 depending on whether the version of the theory considered included supersymmetry or not. More recently it was found that the theory also requires the presence of extended objects of higher dimensional extension. These are called $p$-branes when the spatial extension has $p$ dimensions. Thus point particles and strings have $p$ value 0 and 1 respectively. All these couple to appropriate generalised abelian gauge field analogous to the Maxwell field that couples to point particles.

In this latter situation, Faraday and Maxwell developed the familiar notions of electric charge, electric flux and magnetic flux, all of which are conserved. The topological significance of these ideas was subsequently developed by pure mathematicians, particularly Hodge [3]. Given the physical importance it is natural to seek to enumerate these conserved quantities and establish any interrelations when the propagation of $p$-branes is considered in a fixed background space-time that may have any dimension and any topology (as long as it is oriented).

This is the task Marcos Alvarez and I set ourselves and we found the answer to be unexpectedly rich and complicated, hep-th/0303229.

The "electrically charged" $p$-branes are sources of the generalised gauge field that satisfies a generalised Maxwell equation. The requirement that these equations be integrable

\footnotetext{
${ }^{*}$ Speaker.
} 
imposes selection rules on the allowed configurations of the branes in space-time. This can be illustrated by simple examples involving point particles moving on a two dimensional space-time. Suppose space-time is a two-cylinder. The trajectory of a particle in spacetime constitutes its world-line. If that winds once around the space-time cylinder there is no problem, as the electric lines of force attached to the world-line disappear to infinity at the two ends of the cylinder. If the two ends of the cylinder are joined, thereby forming a two-torus there is a problem as the lines of force clash. This means that the Maxwell equations cannot be integrated and the configuration is forbidden.

In fact this result generalises as Misner and Wheeler (1957) 团 and more generally Henneaux and Teitelboim (1990) [2 have noted: If space-time is closed and Maxwell's equations are integrable, the only allowed brane world-volumes are those that are topologically trivial and so carry zero total conserved charge. The example of the cylinder indicates that in order to obtain a more interesting situation space-times should be considered that are open, and so not closed.

It is convenient to keep the space-time $\mathcal{M}$ compact and this is achieved by imagining it to have a boundary $\mathcal{B}$ :

$$
\partial \mathcal{M}=\mathcal{B}
$$

The main result of our work is that in this more general situation the allowed brane configurations are those whose world-volumes are homologous to cycles on the boundary $\mathcal{B}$ of space-time.

In order to establish this we have to introduce the mathematical theory of relative homology/cohomology applied to space-time. The theory of absolute homology/cohomology is relatively familiar to physicists (see for example, Misner and Wheeler 䧃) and is covered in many textbooks (e.g. A Schwarz [⿹]).

Let us now explain the set up that we assume and the notation. The $p$-brane has a world-volume $w$ that is a $(p+1)-$ cycle, $\partial w=0$.

The geometric term specifying the coupling to a $(p+2)-$ form field strength $F=d A$ is via the term in the action

$$
" q \int_{w} A^{\prime \prime}
$$

This is gauge invariant with respect to $A \rightarrow A+d \chi$ as the alteration is formally $\int_{w} d \chi=$ $\int_{\partial w} \chi=0$, using Stokes' theorem and the closure of $w$. Of course the field strength, $F$, is gauge invariant.

We shall assume Maxwell's equations to have the form

$$
\begin{gathered}
d F=0, \\
d\left(h^{*} F\right)={ }^{*} j .
\end{gathered}
$$

${ }^{*} F$ denotes the Hodge dual of $F$ and so is a $(m-p-2)-$ form. $j$ is the electric current form (so its dual ${ }^{*} j$ has degree $(m-p-1)$ ). The only slightly unexpected feature is the appearance of the scalar function $h$ which can be thought of as an exponentiated dilaton field. Thus it equals unity in the vacuum and is otherwise positive. Such a possibility is 
common to supergravity theories. Irrespective of this it follows from the Maxwell equations that

$$
d^{*} j=0,
$$

expressing the conservation of the current. Initially we do not need to assume an explicit form of $j$ that will be appropriate to geometric branes, and shall introduce it later. Notice that the Maxwell equations on which our analysis is based are gauge invariant as there is no explicit mention of $A$.

We are ready to review the notion of conserved electric charge, given in terms of the current $j$. Tentatively consider

$$
Q(S)=\int_{S}^{*} j,
$$

where $\mathrm{S}$ is a chain of dimension $(m-p-1)$, matching the degree of ${ }^{*} j$.

If $S$ is changed by a homology, $S \rightarrow S^{\prime}=S+\partial R \sim S$,

$$
Q(S)=Q\left(S^{\prime}\right)
$$

as the alteration $\int_{\partial R}{ }^{*} j=\int_{R} d^{*} j=0$, using Stokes' theorem and the conservation of current, $j$. Thus $Q$ is indeed conserved.

The trouble with this is that we ought to assume more about $S$, for example, that $S$ is a cycle so that $S^{\prime}$ is also one automatically. Then our argument shows that $Q(S)$ is defined on homology classes within space-time.

Homology classes can be added and so form an abelian group denoted $H_{m-p-1}(\mathcal{M})$, if, as now, $(m-p-1)$-cycles are considered. There is a technical complication that is quickly removed: The class of the cycle $S$ has finite order, $n$, if $n S$ is a boundary and so homologically trivial. Then $Q(S)=\frac{1}{n} Q(n S)=0$. Such classes are called torsion classes and are consequently irrelevant to our present discussion. They form an invariant subgroup that can be divided out to form the "free" classes

$$
F_{m-p-1}(\mathcal{M})=H_{m-p-1}(\mathcal{M}) / T_{m-p-1}(\mathcal{M})
$$

on which the charges are truly defined, and this group can be thought of lattice whose dimension defines the appropriate Betti number $b_{m-p-1}(\mathcal{M})$.

This picture would be perfectly satisfactory if space-time were closed and gauge field were absent. With gauge fields present the second Maxwell equation implies that

$$
Q(S)=\int_{S}^{*} j=\int_{S} d\left(h^{*} F\right)=\int_{\partial S} h^{*} F=0,
$$

using the fact $S$ is a cycle. This is the unfortunate conclusion, alluded to earlier, that all conserved electric charges vanish if space-time is closed.

As already anticipated, a remedy is to allow space-time to have a boundary, $\mathcal{M}$ because then $S$ can be a relative cycle instead of just an absolute cycle. This means $\partial S=\alpha \in \mathcal{B}$. It is natural to assume that there is no outflow of current through the boundary, that is the restriction of the dual current to the boundary vanishes

$$
\left.{ }^{*} j\right|_{\mathcal{B}}=0
$$


Now the electric charge $Q(S)$ is unchanged by "relative homologies", $S \rightarrow S^{\prime}=S+\partial R+\gamma \sim$ $S$ where $\gamma$ is in the boundary, $\mathcal{B}$. The reason is that $\int_{\partial R}{ }^{*} j$ vanishes by Stokes whereas $\int_{\gamma} * j$ vanishes by the condition that there be no outflow of current.

Now, rather than the groups of absolute homology, it is the relative homology groups $H_{m-p-1}(\mathcal{M}, \mathcal{B})$ that are relevant, or better, the free parts $F_{m-p-1}(\mathcal{M}, \mathcal{B})$, and it is these that classify the conserved electric charges.

Now, taking account of Maxwell's equations

$$
Q(S)=\int_{\partial S} h^{*} F=\int_{\alpha}^{*} F=\Phi_{E}(\alpha)
$$

as $\partial S=\alpha \in \mathcal{B}$ and $\left.h\right|_{\mathcal{B}}=0$ since we suppose the the situation on the boundary of space-time simulates the vacuum as far as concerns the scalar fields on which $h$ depends.

We have introduced the notion of electric flux $\Phi_{E}(\alpha)$ through a cycle $\alpha \in \mathcal{B}$ and this applies even if it is not the boundary of a relative cycle such as $S$. It is easy to check that these electric fluxes are unchanged by homologies of $\alpha$ within $\mathcal{B}$. Thus these fluxes are defined by the homology groups $H_{m-p-2}(\mathcal{B})$ or more accurately the free groups $F_{m-p-2}(\mathcal{B})$.

These are quite different from the relative homology groups that classified electric charges and so we recognise that we must distinguish electric charges from electric fluxes. Indeed not all electric fluxes are electric charges as we see from the above when $\alpha$ is not the boundary of a relative cycle. Furthermore some of the electric charges which are also fluxes vanish. This happens if $\alpha=\partial \beta$ for $\beta \in \mathcal{B}$.

In writing down the Maxwell's equations with which we were working we assumed the absence of any magnetic charge. Nevertheless magnetic fluxes may exist:

$$
\Phi_{M}(u)=\int_{u} F
$$

where $u$ is a bulk cycle. Again these are conserved and, this time, are classified by the free part of the absolute homology groups $F_{p+2}(\mathcal{M})=H_{p+2}(\mathcal{M}) / T_{p+2}(\mathcal{M})$.

The conclusion is that there are three distinct types of conserved quantity, electric charge $Q(S)$, electric flux, $\Phi_{E}(\alpha)$ and magnetic flux, $\Phi_{M}(u)$. These are all classified by homology and correspond to the three types of homology that can be considered on space time $\mathcal{M}$ with a boundary $\mathcal{B}$, namely relative homology, absolute homology on $\mathcal{B}$ and absolute homology in the bulk, $\mathcal{M}$, respectively. Correspondingly there are also three types of de Rham cohomology and these are relevant for ${ }^{*} j,{ }^{*} F$ and $F$ but for details I refer to our paper.

The natural question is as to how these concepts are related. For example, we have seen that all electric charges can be expressed as electric fluxes (and so sometimes have to vanish) whereas not all electric fluxes are expressible as electric charges.

It turns out that there is a intricate but beautiful mathematical structure that corresponds to all such physical interrelations. This concerns the existence of two exact sequences of, of homology and cohomology together with various isomorphisms and duality 
relations. This is indicated by the diagram

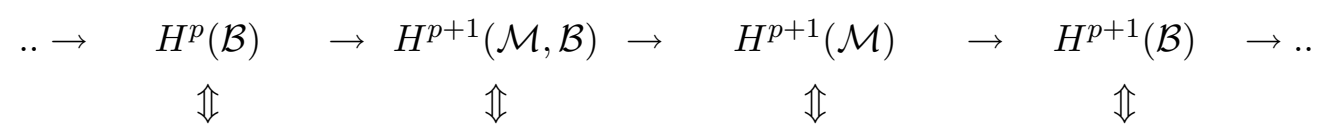

$$
\begin{aligned}
& . . \rightarrow H_{m-p-1}(\mathcal{B}) \rightarrow H_{m-p-1}(\mathcal{M}) \rightarrow H_{m-p-1}(\mathcal{M}, \mathcal{B}) \rightarrow H_{m-p-2}(\mathcal{B}) \rightarrow . .
\end{aligned}
$$

In the second line can be recognised homology groups of the types already mentioned whereas in the first line there appear cohomology groups. The horizontal arrows represent a sequence of group homomorphisms with the property of being exact. This means that each stage the kernel of a homomorphism coincides with the image of the preceding homomorphism. This subgroup that plays the role of being both a kernel and an image is denoted $K$.

The vertical arrows relating homology and cohomology indicate what is called the Poincaré-Lefschetz isomorphism. Notice how the groups so related have complementary degree.

The exact sequences above are finite in each direction as the dimension of spacetime, $m$, is finite. In addition there exist duality relations involving a horizontal flip of the diagram. All these relations play a role in answering the physical question as to the relations between the different sorts of charge and flux, as explained in our paper.

Now let us take account of the fact that the electric charge density we consider is confined to the world volumes of the branes, themselves bulk cycles. This means that ${ }^{*} j(w)$, the dual current form associated with the world-volume, $w$ is distribution-valued. It vanishes off $w$ and its differentials are all tranverse to $w$. It is a closed form whose restriction to the boundary vanishes. Thus it provides certain of the upwards arrows in the vertical (Poincaré-Lefschetz) isomorphisms in the exact sequence diagram above.

Furthermore if we look again at the Maxwell equation, ${ }^{*} j=d\left(h^{*} F\right)$, we see that ${ }^{*} j$ is exact, not just closed (i.e. $j$ conserved). This means that ${ }^{*} j$ belongs to the kernel subgroup of the relevant de Rham cohomology group $\left(K_{\text {deRham }}^{m-p-1}(\mathcal{M}, \mathcal{B})\right)$. The PoincaréLefschetz isomorphism (the vertical double arrow) then implies that the world-volume, $w$, be in its appropriate kernel subgroup, namely $K_{m-p-1}(\mathcal{M})$. This means that $w$ is cohomologous to a cycle on the boundary of space-time. This is one of our main results, the selection rule on allowed world-volumes.

In the brane set-up we are dealing with the electric charges can be evaluated in a fairly explicit way (as Henneaux and Teitelboim [2] noted):

$$
Q(w, S)=\int_{S}^{*} j(w)=q I(w, S)
$$

where $I(w, S)$ is the intersection number between the bulk cycle $w$ and the relative cycle, $S$ (which indeed possess complementary dimensions). Thus the electric charge has a topological interpretation.

Let us choose bases $\left\{w_{i}\right\}$ and $\left\{S_{j}\right\}$ for the two relevant lattices of free homology. Then $I\left(w_{i}, S_{j}\right)$ constitutes a matrix known as the intersection matrix and from it all intersection numbers are calculable. Mathematical consequences of the above exact sequence structure are that this intersection matrix is square and unimodular, so that its determinant, Det $I=$ 
\pm 1 . In particular, the intersection matrix is nonsingular and this property poses a dilemma in view of the earlier result that all electric charges $Q(S)$ vanish when $S$ lies in the kernel of its relative homology group:

$$
Q(w, S)=0 \quad \text { for all } S \in K(\mathcal{M}, \mathcal{B}) .
$$

Thus complete rows of a nonsingular matrix apparently vanish. The first step in resolving this paradox is to note that the vanishing theorem followed from use of Maxwell's equations whereas the evaluation of charges as intersection numbers did not.

The second step is to partition the intersection matrix according to blocks corresponding to the relevant kernel subgroups and their cosets.

$$
I(w, S)={ }_{K(\mathcal{M})}^{(H / K)(\mathcal{M})}\left(\begin{array}{cc}
A & Y(\mathcal{M}, \mathcal{B}) \\
& (H / K)(\mathcal{M}, \mathcal{B}) \\
X & B
\end{array}\right) .
$$

The third step is to understand that $I(K(\mathcal{M}), \mathcal{K}(\mathcal{M}, \mathcal{B}))$ vanishes, as can be checked explicitly. In other words, in the intersection matrix above the submatrix $X$ vanishes. The diagonal block submatrices $A$ and $B$ are square and hence $\pm 1=\operatorname{Det} I=\operatorname{Det} A \operatorname{Det} B$ so that both submatrices $A$ and $B$ are unimodular. We see that the electric charges $Q(w, S)$ vanish for $S$ in the appropriate kernel only if the world-volume $w$ is in its appropriate kernel. This means that the Maxwell equations are only integrable for word-volumes $w$ in the kernel subgroup of the absolute homology group, since otherwise the non-vanishing nature of the electric charge would contradict the consequence of integrating Maxwell's equations as seen above. Thus we conclude that Maxwell's equations can only be integrable if the brane world-volume lies in the kernel, thereby confirming the conclusion previously derived from the Poincaré-Lefschetz isomorphism.

Thus we have arrived at a perfectly consistent picture of the notions of electric charges and fluxes and their interrelations which is intimately related to the mathematical structures described, namely the exact sequences and a rather precise structure displayed by the intersection matrix between relative cycles and absolute bulk cycles.

As already mentioned, magnetic charges have been excluded from the scenario considered but magnetic fluxes are nevertheless possible. It turns out that they are indeed quantised (à la Dirac [1]) in terms of of inverse units of the electric charge constant, $q$. So electric charge is also quantised but electric flux is not necessarily so when it cannot be equated to an electric charge.

Magnetic fluxes are associated with absolute bulk cycles of the appropriate dimension, $(p+2)$. Included amongst these cycles are ones contained in the boundary, $\mathcal{B}$, of spacetime and through these there may also be an electric flux of a field strength of degree $(m-p-2)$. This situation is particularly interesting. If such a cycle is in the kernel subgroup of $H_{p+2}(\mathcal{B})$, the magnetic flux through it vanishes while the electric flux, being equal to an electric charge, is quantised. On the other hand for the remaining cycles, not 
in the kernel, the magnetic flux need not vanish but is quantised, while the electric flux need not be quantised. This phenomenon is intriguingly reminiscent of dyonic behaviour.

Finally I would like to say that all this beautiful mathematical structure captures only part of that which exists in the superstring theories, many of whose ingredients have been ignored in order to simplify our treatment. Obviously an important future step would be incorporate more of these features.

\section{References}

[1] PAM Dirac, "Quantised singularities in the electromagnetic field", Proc. Roy. Soc. A133 (1931) 60-72.

[2] M Henneaux and C Teitelboim, "p-form Electrodynamics", Found Phys 16 (1986) 593-717.

[3] WVD Hodge, "The theory and applications of harmonic integrals", CUP 1952.

[4] CW Misner and JA Wheeler; "Classical Physics as Geometry", Annals of Phys. 2 (1957) 525-603.

[5] A Schwarz; "Topology for Physicists", Berlin-Heidelberg-New York; Springer, 1994. 